\title{
What if the LHC does not find supersymmetry in the $\sqrt{s}=7$ TeV run?
}

\author{
Philip Bechtle and Björn Sarrazin \\ Deutsches Elektronen-Synchrotron DESY, Notkestraße 85, D-22607 Hamburg, Germany \\ Klaus Desch, Herbi K. Dreiner, and Peter Wienemann \\ Physikalisches Institut der Universität Bonn, Nussallee 12, D-53115 Bonn, Germany \\ Michael Krämer and Carsten Robens \\ Institute for Theoretical Particle Physics and Cosmology, \\ RWTH Aachen University, D-52056 Aachen, Germany \\ Ben O'Leary \\ Institute for Theoretical Physics and Astrophysics, \\ Würzburg University, Am Hubland, D-97074 Würzburg, Germany
}

\begin{abstract}
We investigate the implications for supersymmetry from an assumed absence of any signal in the first period of LHC data taking at $7 \mathrm{TeV}$ center-of-mass energy and with 1 to $7 \mathrm{fb}^{-1}$ of integrated luminosity. We consider the zero-lepton plus four jets and missing transverse energy signature, and perform a combined fit of low-energy measurements, the dark matter relic density constraint and potential LHC exclusions within a minimal supergravity model. A non-observation of supersymmetry in the first period of LHC data taking would still allow for an acceptable description of low-energy data and the dark matter relic density in terms of minimal supergravity models, but would exclude squarks and gluinos with masses below $1 \mathrm{TeV}$.
\end{abstract}

\section{INTRODUCTION}

The Higgs sector of the Standard Model (SM) of elementary particle physics suffers from the hierarchy problem between the weak scale and the Planck scale. Extending the SM through supersymmetry (SUSY) is a very promising solution [1]. The new supersymmetric particles must then have masses of order $1 \mathrm{TeV}$. Furthermore, supersymmetric particles can contribute to low-energy observables via radiative quantum corrections. In fact, SUSY models provide an excellent fit to the extensive data, see for example Refs. 2- 6]. The fits generically prefer a light SUSY spectrum, typically below $1 \mathrm{TeV}$.

The ATLAS and CMS experiments at the Large Hadron Collider (LHC) at CERN have so far analyzed $35 \mathrm{pb}^{-1}$ of integrated luminosity at $7 \mathrm{TeV}$ centerof-mass energy. A total integrated luminosity of 1 to $7 \mathrm{fb}^{-1}$ can realistically be achieved at this energy through 2012. These data will significantly extend the search reach for SUSY 7, 8].

The minimal supersymmetric SM has 124 free parameters. However, current precision observables and direct search limits only provide sensitivity to very restricted SUSY models with a small number of parameters, like minimal supergravity (mSUGRA) [1] which only has 5 free parameters beyond those of the SM. In the following, we thus focus on mSUGRA models. Specifically, they are characterized by a common supersymmetric scalar mass $M_{0}$, a common gaugino mass $M_{1 / 2}$, a universal trilinear coupling $A_{0}$, the ratio of the two Higgs vacuum expectation values, $\tan \beta$, and the sign of the Higgs mixing mass parameter, $\operatorname{sign}(\mu)$.

At the LHC, supersymmetry can be searched for in channels with jets, leptons and missing transverse energy [21]. Studies by ATLAS and CMS reveal that squarks and gluinos with masses up to about $700 \mathrm{GeV}$ could be discovered at $7 \mathrm{TeV}$ energy and $1 \mathrm{fb}^{-1}$ integrated luminosity [7, 8]. If no signal is found, one can significantly constrain supersymmetric models and exclude squarks and gluinos with masses close to $1 \mathrm{TeV}$.

To obtain consistent limits on the SUSY parameter space and the resulting mass spectrum in the absence of a SUSY signal at the LHC, one needs to combine potential LHC exclusion limits and current low energy precision observables in a global fit. In the following we employ the FitTino framework [6, 9] to study such a scenario.

\section{FIT OBSERVABLES}

We follow the FitTino analysis in Ref. 6] and consider the following set of low-energy observables and existing collider limits in light of the mSUGRA model: (i) rare decays of B- and K-mesons; (ii) the anomalous magnetic moment of the muon, $a_{\mu}$; (iii) electroweak precision measurements from LEP, SLC and the Tevatron and the Higgs boson mass limit from LEP; and (iv) the relic density of cold dark matter in the universe, $\Omega_{\chi}$. In contrast to Ref. [6], we employ the program HiggsBounds [10] and not a rigid Higgs mass 
limit. We refer to Ref. [6] for a detailed discussion of the low-energy inputs and the collider limits.

At the LHC, the most stringent limits on supersymmetric models with $R$-parity conservation can be expected from searches in channels with jets, leptons and missing transverse energy, $E_{T}^{\text {miss }}$. We follow the analysis presented in Ref. [7] and consider the search channel with four jets, zero leptons and $E_{T}^{\text {miss }}$. This channel drives the sensitivity, in particular for large $M_{1 / 2}$. The selection cuts are

- four or more central jets with the pseudorapidity $\mid \eta($ jet $) \mid<2.5$, and with the transverse momentum $p_{T}>100 \mathrm{GeV}$ for the leading jet, and $p_{T}>40 \mathrm{GeV}$ for the other jets;

- an opening angle between the transverse momentum of the three leading jets and $\vec{p}_{T}^{\text {miss }}$ satisfying $\Delta \phi\left(\vec{p}_{T}^{\mathrm{jet}, i}, \vec{p}_{T}^{\mathrm{miss}}\right)>0.2$;

- the missing transverse energy $E_{T}^{\text {miss }}>80 \mathrm{GeV}$;

- the ratio of the missing transverse energy and the effective mass satisfying $E_{T}^{\text {miss }} / M_{\text {eff }}>0.2$;

- the transverse sphericity [11] $S_{T}>0.2$;

- no leptons with $p_{T}>20 \mathrm{GeV}$.

The effective mass is defined as the scalar sum of the transverse momenta of all main objects, i.e.

$$
M_{\mathrm{eff}}=\sum_{i=1}^{N_{\mathrm{jets}}=4} p_{T}^{\mathrm{jet}, i}+E_{T}^{\mathrm{miss}} .
$$

The SM background processes have been described in detail in Ref. [7]. After the cuts listed above, the combined SM cross section has been estimated to $\sigma_{\mathrm{SM}}=2.42 \mathrm{pb}$ at $7 \mathrm{TeV}$. The signal cross section is dominated by squark and gluino pair production, $p p \rightarrow \tilde{q} \tilde{q}^{*}, \tilde{q} \tilde{q}, \tilde{q} \tilde{g}$ and $\tilde{g} \tilde{g}$, but all SUSY pair production processes are included in our numerical analysis. We use HerwiG++ [12] in combination with the parameterised fast detector simulation Delphes [13] to obtain the detector response and, in particular, the shape of the $M_{\text {eff }}$ distribution for a given point in the supersymmetric parameter space [22]. The signal estimate is normalized to the NLO+NLL QCD prediction for the inclusive squark and gluino cross sections [14].

To obtain good sensitivity to a SUSY signal, the full distribution of $M_{\text {eff }}$ is included in the statistical analysis. We consider ten bins in the range $0<M_{\text {eff }}<$ $4 \mathrm{TeV}$ and calculate the $\chi^{2}$ contribution to the SUSY parameter fit from the number of signal and background events in each bin of the $M_{\text {eff }}$ distribution. We define a test statistic $t=-2 \ln Q$ with $Q$ being the likelihood ratio

$$
Q=\prod_{i=1}^{N_{\mathrm{bins}}} \frac{\mathcal{L}\left(\mu_{i}=s_{i}+b_{i} ; n_{i}\right)}{\mathcal{L}\left(\mu_{i}=b_{i} ; n_{i}\right)} .
$$

Here $\mathcal{L}(\mu ; n)=\mu^{n} e^{-\mu} / n$ ! is the Poisson probability to observe $n$ events if $\mu$ are expected. $s_{i}$ and $b_{i}$ are the expected number of signal and background events in bin $i$, and $n_{i}$ is the observed event count in this bin. $s_{i}$ is a function of the SUSY parameters, whereas $b_{i}$ is fixed. We consider a signal excluded with $95 \%$ confidence level (CL) if

$$
\mathrm{CL}_{s+b}=\int_{t_{\mathrm{obs}}}^{\infty} P_{s+b}(t) d t<0.05 .
$$

Here $P_{s+b}(t)$ is the probability density function of $t$ assuming the presence of a signal and $t_{\mathrm{obs}}$ the actually observed value of $t$. Uncertainties on the cross-sections are taken into account by a correlated smearing of the expected event numbers. A given $\mathrm{CL}_{s+b}$ value can be approximately translated into a $\chi^{2}$ contribution using the formula 15]

$$
\chi^{2}=2\left[\operatorname{erf}^{-1}\left(1-2 \mathrm{CL}_{s+b}\right)\right]^{2} .
$$

To obtain expected exclusion limits we use the Asimov data set $n_{i}=b_{i}, i=1, \ldots, N_{\text {bins }}$.

\section{NUMERICAL RESULTS}

We present results from a global fit of the mSUGRA model to low-energy precision observables, the dark matter relic density, existing collider data from LEP, SLC and the Tevatron, and potential LHC exclusion limits. We consider LHC scenarios corresponding to $35 \mathrm{pb}^{-1}$, and 1,2 and $7 \mathrm{fb}^{-1}$ integrated luminosity.

In Fig.1 we show the mSUGRA parameter region in $M_{0}$ and $M_{1 / 2}$ compatible with the existing low energy observables, the existing collider limits from LEP, SLC and the Tevatron, and the cold dark matter relic density, but no LHC exclusions imposed. Note that a positive sign of $\mu$ is preferred to describe the anomalous magnetic moment of the muon, so we have fixed $\operatorname{sign}(\mu)=+$ and determined $M_{0}, M_{1 / 2}, A_{0}$, and $\tan \beta$ from the fit. Including the $1 \sigma$ uncertainty, we find $M_{0}=75_{-29}^{+115} \mathrm{GeV}, M_{1 / 2}=329_{-83}^{+92} \mathrm{GeV}$, $A_{0}=417_{-725}^{+715} \mathrm{GeV}$ and $\tan \beta=13_{-7}^{+10}$, in good agreement with Ref. [6]. The minimum $\chi^{2}$ value is 19 for 20 degrees of freedom. Note that in regions with small $M_{0}$ and large $M_{1 / 2}$ the $\tilde{\tau}$ may be the lightest supersymmetric particle; such regions are thus excluded from the fit. In Fig.1 we also show our estimate of the LHC exclusion limits in the four-jet, zero-lepton and missing transverse energy channel for different integrated luminosities. The area below the curves can be excluded at 95\% CL. We assign 30\% systematic uncertainty to the SUSY signal cross-section, and $20 \%$ systematic uncertainty to the background event rate in the signalenriched region, which is dominantly electroweak [7]. The LHC signal estimate for the four jets, zero leptons and $E_{T}^{\text {miss }}$ channel is insensitive to variations in $\tan \beta$ and $A_{0}$ within the systematic uncertainty. Our 


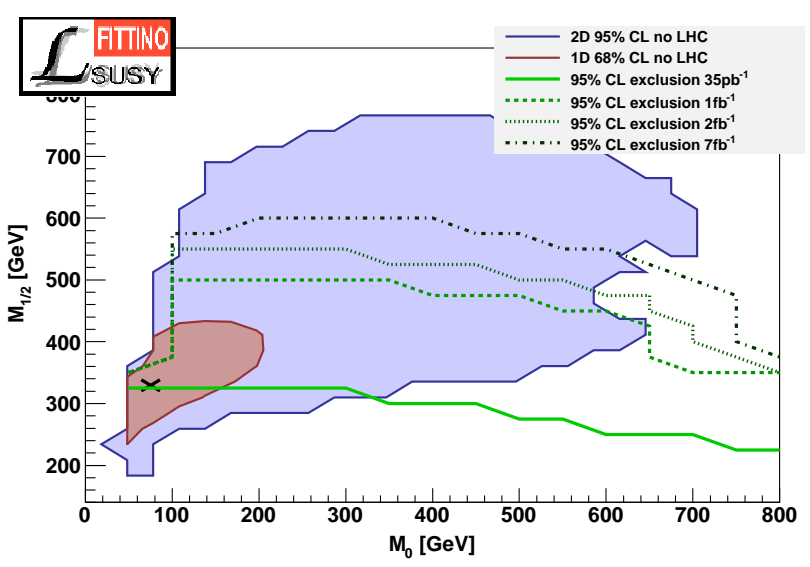

FIG. 1: mSUGRA parameter region in $M_{0}$ and $M_{1 / 2}$ compatible with low-energy observables, current collider data from LEP, SLC and the Tevatron, and the dark matter relic density. Shown are the two-dimensional $95 \%$ and onedimensional $68 \%$ CL regions. Also shown is our estimate of the potential LHC 95\% CL exclusion limits in the fourjet, zero-lepton and $E_{T}^{\text {miss }}$ channel for different integrated luminosities.

results are in good agreement with current LHC limits at $35 \mathrm{pb}^{-1}[16$, 17], and with the projected ATLAS discovery potential at higher luminosities [7], bearing in mind that we use an improved signal estimate including the NLO+NLL QCD corrections.

We now combine the potential LHC exclusion limits, the current low-energy precision and collider observables, and the dark matter relic density constraint in a global fit. We assume $2 \mathrm{fb}^{-1}$ integrated luminosity as our base scenario, but also discuss the impact of the LHC exclusions at $35 \mathrm{pb}^{-1}$, and at 1 and $7 \mathrm{fb}^{-1}$ below. The result of our combined mSUGRA fit assuming no SUSY signal at the LHC with $2 \mathrm{fb}^{-1}$ is shown in Fig.2 The best fit now corresponds to $M_{0}=270_{-143}^{+423} \mathrm{GeV}, M_{1 / 2}=655_{-81}^{+150} \mathrm{GeV}, A_{0}=$ $763_{-879}^{+1238} \mathrm{GeV}$ and $\tan \beta=32_{-21}^{+18}$, with a minimum $\chi^{2}$ value of 24 for 21 degrees of freedom. The corresponding sparticle mass spectrum is presented in Fig. 3 and features most probable squark and gluino masses beyond $1 \mathrm{TeV}$.

An LHC exclusion in the zero-lepton, four-jet plus $E_{T}^{\text {miss }}$ channel is mainly sensitive to the squark and gluino masses and would drive $M_{0}$ and $M_{1 / 2}$ to larger values. The low-energy precision observables and the relic density, on the other hand, are mainly constraining the masses of colour-neutral sparticles. Supersymmetric models with common scalar and gaugino masses like mSUGRA connect these two, leading to a tension between the two sets of observables. In addition, for larger $M_{0}$ and $M_{1 / 2}$ both $a_{\mu}$ and $\Omega_{\chi}$ require an increased $\tan \beta$. It is also noteworthy that the global fit allows areas in the SUSY parameter space at $95 \% \mathrm{CL}$, which are located in the

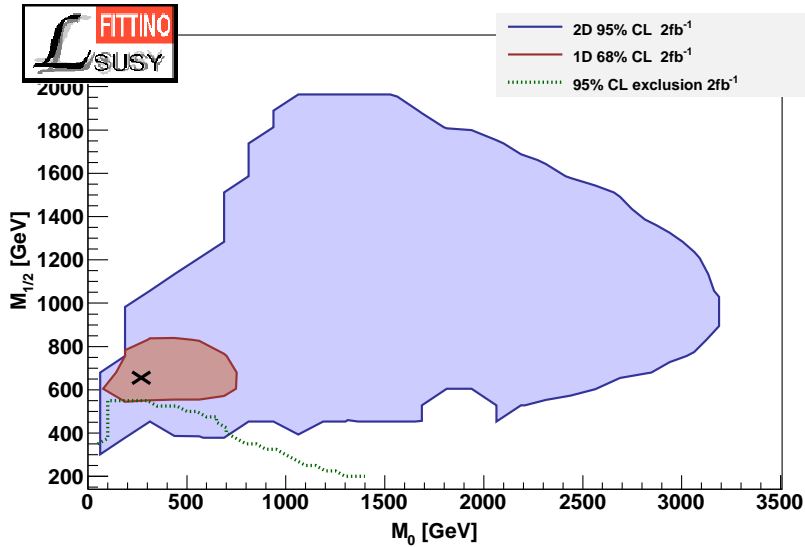

FIG. 2: mSUGRA parameter region in $M_{0}$ and $M_{1 / 2}$ compatible with low-energy observables, current collider data from LEP, SLC and the Tevatron, the dark matter relic density, and a potential LHC exclusion limit in the fourjet, zero-lepton and $E_{T}^{\text {miss }}$ channel for $2 \mathrm{fb}^{-1}$ integrated luminosity. Also shown is the the potential LHC 95\% CL exclusion limit.

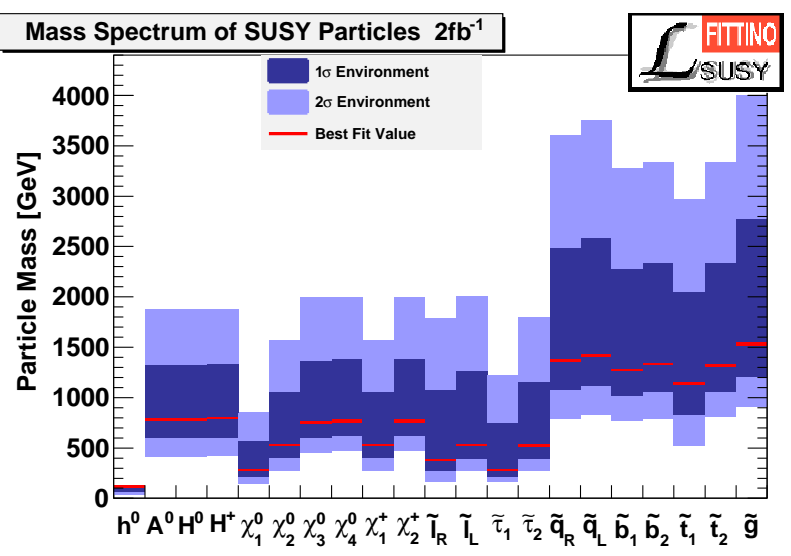

FIG. 3: SUSY mass spectrum as predicted by a combined mSUGRA fit of low-energy observables, current collider data from LEP, SLC and the Tevatron, the dark matter relic density, and a potential LHC exclusion limit in the four-jet, zero-lepton and $E_{T}^{\text {miss }}$ channel for $2 \mathrm{fb}^{-1}$ integrated luminosity.

region of $95 \%$ CL exclusion of the LHC, see Fig.2. This is due to the weak dependence of the LHC contribution to the $\chi^{2}$ on $M_{1 / 2}$. Furthermore values of $M_{0}$ and $M_{1 / 2}$ below the direct LHC limit allow for a significantly better $\chi^{2}$ from the low energy data, compensating the contribution from the LHC. Thus the lower limits on the SUSY masses from the global fit including the LHC are significantly lower than the direct exclusion limits.

Fig. 1 presents the impact of the LHC exclusions on the $\tilde{q}_{R}$ and $\tilde{l}_{R}$ mass spectrum from the global mSUGRA fit, assuming $35 \mathrm{pb}^{-1}$, and 1, 2 and $7 \mathrm{fb}^{-1}$. Already with $1 \mathrm{fb}^{-1}$ the LHC exclusion would push the lower limit on the squark mass above the $\mathrm{TeV}$ - 

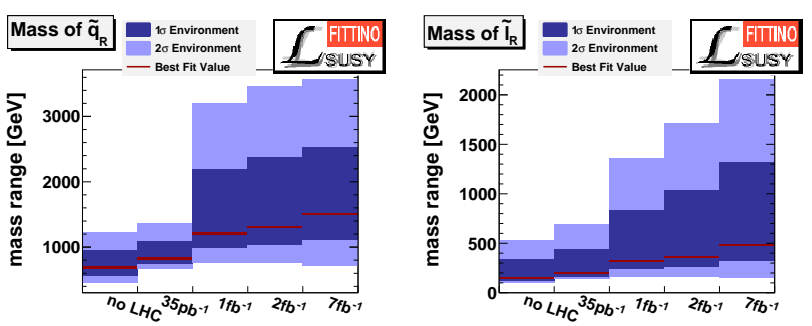

FIG. 4: $\tilde{q}_{R}$ and $\tilde{l}_{R}$ masses as predicted by a combined mSUGRA fit of low-energy observables, current collider data from LEP, SLC and the Tevatron, the dark matter relic density, and a potential LHC exclusion limit in the four-jet, zero-lepton and $E_{T}^{\text {miss }}$ channel for $35 \mathrm{pb}^{-1}$, and 1 , 2 and $7 \mathrm{fb}^{-1}$ integrated luminosity.

scale. The $\tilde{l}_{R}$ mass, which is mainly determined from the low-energy observables, is predicted in the range between $300 \mathrm{GeV}$ and $500 \mathrm{GeV}$. Note that a combined mSUGRA fit without the constraints on $a_{\mu}$ and $\Omega_{\chi}$ results in very large sparticle mass uncertainties.

\section{CONCLUSIONS}

We have presented a first global mSUGRA analysis of supersymmetric models which includes the current low-energy precision measurements, the dark matter relic density as well as potential LHC exclusion limits from direct SUSY searches in the zero-lepton plus jets and missing transverse energy channel.

We conclude that it is, in principle, possible to reconcile the supersymmetric description of low-energy observables and the dark matter relic density with a non-observation of supersymmetry in the first phase of the LHC, despite some tension building up in a combined fit within the mSUGRA framework. Moreover, we find that a global mSUGRA fit including potential LHC exclusion limits would yield lower bounds on squark and gluino masses of about $1 \mathrm{TeV}$ already with $1 \mathrm{fb}^{-1}$ integrated LHC luminosity.

While our study is exploratory in the sense that it is based on one search channel only, and on a simplified description of the LHC detectors, it clearly demonstrates the potential of the first phase of LHC running at $7 \mathrm{TeV}$ in 2011/12 to constrain supersymmetric models and the sparticle mass spectrum.

\section{Acknowledgments}

We thank Sascha Caron and Werner Porod for valuable discussions. This work has been supported in part by the Helmholtz Alliance "Physics at the Terascale", the DFG SFB/TR9 "Computational Particle Physics", the DFG SFB 676 "Particles, Strings and the Early Universe", the European Community's
Marie-Curie Research Training Network under contract MRTN-CT-2006-035505 "Tools and Precision Calculations for Physics Discoveries at Colliders" and the Helmholtz Young Investigator Grant VH-NG-303. MK thanks the CERN TH unit for hospitality.

[1] H. P. Nilles, Phys. Rept. 110 (1984) 1.

[2] R. R. de Austri, R. Trotta, L. Roszkowski, JHEP 0605 (2006) 002.

[3] B. C. Allanach, K. Cranmer, C. G. Lester et al., JHEP 0708 (2007) 023.

[4] R. Lafaye, T. Plehn, M. Rauch et al., Eur. Phys. J. C54 (2008) 617-644.

[5] O. Buchmueller et al., JHEP 0809 (2008) 117.

[6] P. Bechtle, K. Desch, M. Uhlenbrock and P. Wienemann, Eur. Phys. J. C 66 (2010) 215.

[7] The ATLAS collaboration, "Prospects for Supersymmetry discovery based on inclusive searches at a 7 TeV centre-of-mass energy with the ATLAS detector," ATL-PHYS-PUB-2010-010.

[8] The CMS Collaboration, "Performance of Methods for Data-Driven background Estimation in SUSY Searches", CMS-PAS-SUS-10-001.

[9] P. Bechtle, K. Desch and P. Wienemann, Comput. Phys. Commun. 174 (2006) 47.

[10] P. Bechtle, O. Brein, S. Heinemeyer, G. Weiglein and K. E. Williams, Comput. Phys. Commun. 181 (2010) 138.

[11] The ATLAS collaboration, "Searching for Supersymmetry with two same-sign leptons, multi-jets plus missing transverse energy in ATLAS at $\sqrt{s}=10 \mathrm{TeV}$, ATL-PHYS-PUB-2009-085, 2009.

[12] M. Bahr et al., Eur. Phys. J. C 58 (2008) 639.

[13] S. Ovyn, X. Rouby and V. Lemaitre, arXiv:0903.2225 [hep-ph].

[14] W. Beenakker, R. Höpker, M. Spira et al., Nucl. Phys. B492 (1997) 51-103; W. Beenakker, M. Krämer, T. Plehn et al., Nucl. Phys. B515, 3-14 (1998); A. Kulesza, L. Motyka, Phys. Rev. Lett. 102 (2009) 111802 and Phys. Rev. D80 (2009) 095004; W. Beenakker, S. Brensing, M. Krämer et al., JHEP 0912 (2009) 041 and JHEP 1008 (2010) 098.

[15] J. R. Ellis, S. Heinemeyer, K. A. Olive, A. M. Weber, G. Weiglein, JHEP 0708 (2007) 083.

[16] V. Khachatryan et al. [CMS Collaboration], arXiv:1101.1628 [hep-ex]].

[17] Armir Farbin, for the ATLAS Collaboration, talk at the conference "New data from the Energy Frontier," Aspen, 13-18 February 2011.

[18] H. Dreiner, C. Luhn and M. Thormeier, Phys. Rev. D 73 (2006) 075007.

[19] H. Dreiner, G. Ross, Nucl. Phys. B 365 (1991) 597.

[20] H. Dreiner, M. Krämer, J. M. Lindert and B. O'Leary, JHEP 1004 (2010) 109.

[21] We restrict ourselves to conserved R-parity [1] or proton hexality [18]. R-parity violation leads to diluted or no missing transverse energy [19].

[22] A fast implementation of the four-jet signal calculation using the methods introduced in Ref. [20] is work in progress. 